\title{
Optimize Highly Efficient Genetic Transformation Method of Spring Wheat (Triticum aestivum L.) Cultivars Using Mature Embryo \\ Dongjin $\mathrm{Kim}^{1,2, *}$ and Megan Hager ${ }^{1,3}$
}

\author{
1Department of Plant and Plant Pathology, Montana State University, Bozeman, MT, USA; ${ }^{2}$ Department \\ of Chemistry and Biochemistry, Montana State University, Bozeman, MT, USA; ${ }^{3}$ Department of Land \\ Resources and Environmental Sciences, Montana State University, Bozeman, MT, USA \\ *For correspondence: dongjin.kim@montana.edu
}

[Abstract] Genetic engineering is a powerful tool to develop desired traits in crop plants to make the crops suitable for future demand and changing environments. For the genetic engineering of wheat cultivars, the development of efficient transformation and regeneration systems has always been a primary requirement. Immature embryos have been used as explants for callus generation and genetic transformation of wheat cultivars, but the availability of healthy immature embryos as an explant throughout the year is difficult. In contrast, mature embryos are relatively easy to use throughout the year. The following protocol describes the genetic transformation of wheat cultivars using a mature embryo as explants by the Agrobacterium-mediated transformation method.

Keywords: Wheat, Triticum aestivum, Transformation, Agrobacterium, Mature embryo

[Background] Genetic transformation is the most important technique used in wheat molecular genetics and improvement through genetic engineering. Microparticle bombardment and Agrobacteriummediated transformation are the two most widely used methods for genetic transformation in wheat (Vasil et al., 1992; Ding et al., 2009). Agrobacterium-mediated transformation is a simple, low cost and highly efficient method compared to the direct gene delivery method. Hexaploid wheat possesses a large genome and shows a low rate of regeneration and transformation (Bhalla, 2006). Well-defined tissue culture procedures are needed for callus induction and regeneration for successful genetic engineering in crop plants. Immature embryos, mature embryos, and mature seedlings are used as explant sources. Among these, the most common source of explants is immature embryos due to their high transformation and regeneration efficiency. However, the wheat immature embryos are not readily available throughout the year, and there is a small window where the development stage of the immature embryo can be used for tissue culture (Alikina et al., 2016). When mature embryos are used as explants, they can be collected in large quantities and stored or used immediately. In this study, we used mature embryos from Montana wheat cultivars in order to optimize the protocol for the regeneration of callus and transformation using Agrobacterium-mediated methods. 


\section{Materials and Reagents}

1. $50 \mathrm{ml}$ flask (Fisher Scientific, catalog number: 10-040C)

2. $500 \mathrm{ml}$ flask (PYREX, catalog number: 4980500)

3. Petri dish (Fisher Scientific, catalog number: FB0875713)

4. Wheat seeds

Triticum aestivum L. cv Chinese Spring

Triticum aestivum L. cv Amidon

Triticum aestivum L. cv Choteau

5. Agrobacterium tumefaciens strain LBA4404

6. Rifampicin (Research Products International Corp, catalog number: R640001)

7. Streptomycin (Fisher BioReagents, catalog number: BP910-50)

8. Kanamycin (Fisher BioREagents, catalog number: BP906-5)

9. pGFPGUSPlus (Addgene, Plasmid, catalog number: 64401)

10. D-Mannitol (Sigma-Aldrich, catalog number: M4125)

11. L-glutamic acid (Spectrum Chemical, catalog number: GL125)

12. Yeast extract (Caisson Labs, catalog number: Y001)

13. Tryptone (Research Products International, catalog number: 60060)

14. $\mathrm{KH}_{2} \mathrm{PO}_{4}$ (Sigma-Aldrich, catalog number: 1551139)

15. $\mathrm{NaCl}$ (Sigma-Aldrich, catalog number: S9625)

16. $\mathrm{MgSO}_{4} \cdot 7 \mathrm{H}_{2} \mathrm{O}$ (Sigma-Aldrich, catalog number: $\mathrm{M} 2773$ )

17. MS basal salt (Bioworld, catalog number: $30630070-1$ )

18. D-Maltose (Caisson labs, catalog number: M004)

19. Casein hydrolysate (Sigma-Aldrich, catalog number: 22090)

20. Myo-inositol (Caisson labs, catalog number: M014)

21. L-Proline (Caisson labs, catalog number: P015)

22. Thiamine $\mathrm{HCl}$ (Caisson labs, catalog number: T009)

23. Copper(II) sulfate (Sigma-Aldrich, catalog number: C1297)

24. 2,4-Dichlorophenoxyacetic Acid (2,4-D) (Caisson labs, catalog number: D001)

25. X-Gluc (5-bromo-4-chloro-3-indolyl- $\beta$-D-glucuronide) (GoldBio, catalog number: G1281)

26. Phytagel (Bioworld, catalog number: 40000035)

27. $\mathrm{KCl}$ (Sigma-Aldrich, catalog number: P9541)

28. Acetosyringone (Bioworld, catalog number: 40100297)

29. Timentin (Caisson labs, catalog number: T034)

30. Hygromycin (Spectrum Chemical, catalog number: H3399)

31. Indole-3-acetic Acid (IAA) (Caisson labs, catalog number: 1001)

32. 6-Benzylaminopurine (6-BAP) (Caisson labs, B001)

33. Kinetin Hydrochloride (Spectrum Chemical, catalog number: K3010)

34. Lipoic Acid (spectrum Chemical, catalog number: L1515) 
35. Ethanol (Sigma-Aldrich, catalog number: 16015)

36. Bleach (Clorox, catalog number: 65951360)

37. MG media (see Recipes)

38. Agrobacterium resuspension medium (see Recipes)

39. Co-cultivation media (see Recipes)

40. Callus generation media (see Recipes)

41. Resting media (see Recipes)

42. Selection media (see Recipes)

43. Regeneration Media (see Recipes)

\section{Equipment}

1. Zeiss AxioScope A1 (Carl Zeiss Microscopy, LLC USA)

2. Plant growth chamber (Thermo Scientific, Precision 818)

3. Laminar airflow hood (Thermo Scientific, 1300 Series)

4. Centrifuge (Eppendorf, 5804R)

5. Shaker incubator (IKA works KS 4000i)

6. Spectrophotometer (Eppendorf BioPhotometer, model: 6131)

7. Camera (Canon EOS Rebel T6)

\section{Software}

1. ImageJ (NIH Image, webpage: imagej.net/ImageJ)

\section{Procedure}

A. Seed sterilization

1. Select healthy seeds and soak in sterile distilled water for $30 \mathrm{~min}$ (after these steps are done in laminar airflow hood).

2. Treat with $70 \%$ ethanol $(\mathrm{v} / \mathrm{v})$ for $5 \mathrm{~min}$, and wash with sterile distilled water for three times.

3. Sterile seed with $30 \%(\mathrm{v} / \mathrm{v})$ commercial bleach in shaking incubator at room temperature for 30 $\min$.

4. Finally, wash seeds 5-6 times with sterile water.

B. Mature embryo preparation and callus induction

1. After surface sterilization, germinate seeds overnight on moist filter paper at $25^{\circ} \mathrm{C}$ in the dark.

2. Excise the embryos from the endosperm using forceps and a fine scalpel under a laminar hood.

3. Place the $20-30$ embryos scutellum facing up on the callus generation media.

4. Incubate the embryos at $25^{\circ} \mathrm{C}$ in the dark for 2 weeks. 
5. After two weeks, transfer only embryogenic callus to fresh callus regeneration media.

6. Keep the embryogenic callus at $25{ }^{\circ} \mathrm{C}$ incubator in the dark for 4 weeks prior to transformation.

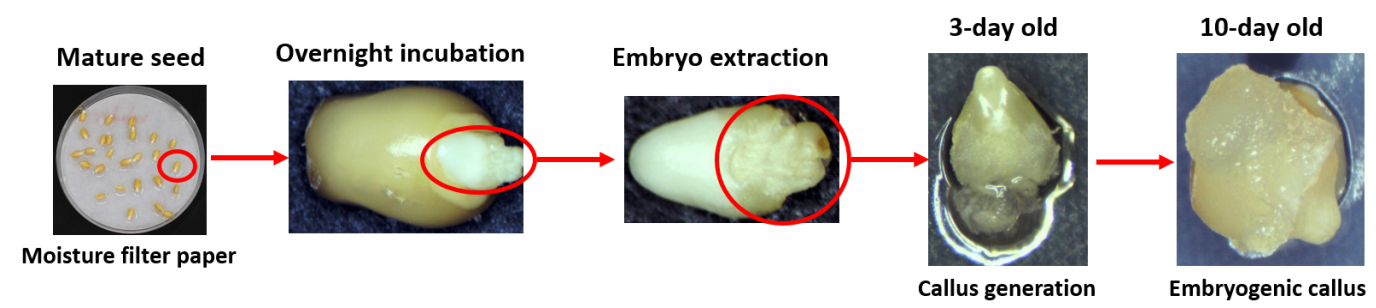

Figure 1. Callus generation step from mature seed. Over-night incubation of mature seeds on moist filter paper at $25^{\circ} \mathrm{C}$ incubator in the dark. The next day, extract embryo using forceps and a fine scalpel under a laminar hood. Place the embryos on the callus generation media and incubate in the dark at $25^{\circ} \mathrm{C}$. Observed callus after 3 days on the callus generation media, and separate embryogenic callus from the embryo after 10 days.

C. Preparation of Agrobacterium cells and co-cultivation with wheat callus

1. Incubate a single colony of Agrobacterium strain LBA4404 in $10 \mathrm{ml} \mathrm{MG}$ media supplemented with Rifampicin, Streptomycin and Kanamycin in $50 \mathrm{ml}$ flask overnight at $28{ }^{\circ} \mathrm{C}$ on shaking incubator at $250 \mathrm{rpm}$.

2. The next day, add $1 \%$ of the primary culture to a $500 \mathrm{ml}$ flask containing $100 \mathrm{ml} \mathrm{MG}$ medium.

3. Cultivate the cells to reach $\mathrm{OD}_{600}>1$ (optical density). Normally, it takes $16-18 \mathrm{~h}$.

4. Pellet the Agrobacterium culture at $12,000 \mathrm{rpm}$ for $15 \mathrm{~min}$ at $4{ }^{\circ} \mathrm{C}$.

5. Resuspend the pellet with the Agrobacterium resuspension medium to adjust the $\mathrm{OD}_{600}$ to 0.3 .

6. Place the 4-week-old 25-30 embryogenic callus into a sterile Petri dish.

7. Pour the $100 \mu \mathrm{l}$ of resuspended Agrobacterium on the embryogenic calli, and incubate the Agrobacterium on the calli for $5 \mathrm{~min}$.

8. Transfer the inoculated calli to sterile filter paper and dry for $5 \mathrm{~min}$.

9. Transfer the calli to the co-cultivation medium and culture the callus for 3 days at $24{ }^{\circ} \mathrm{C}$ in the dark.

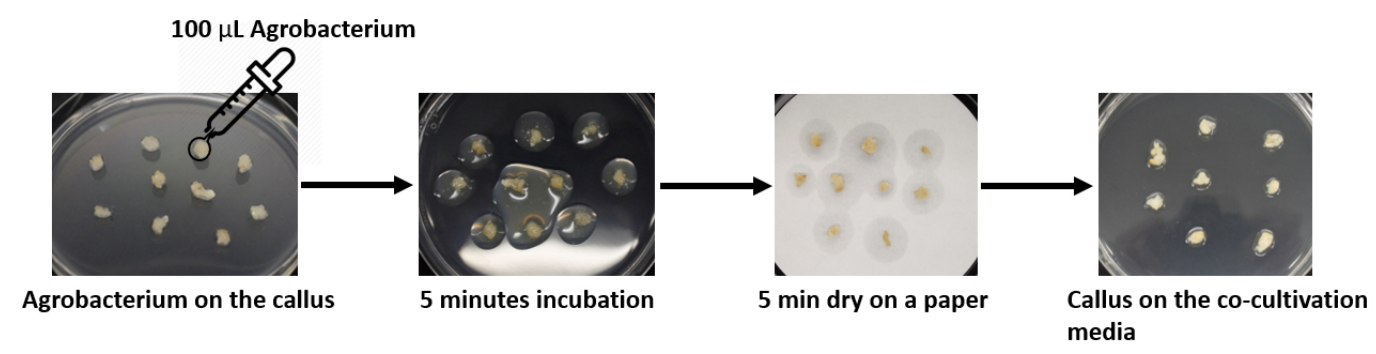

Figure 2. The co-cultivation procedure of the embryogenic callus and Agrobacterium.

Pour the $100 \mu \mathrm{l}$ resuspended Agrobacterium on the 4-week-old embryogenic calli and incubation the callus for $5 \mathrm{~min}$. Transfer the callus on the sterilized filter paper and dry $5 \mathrm{~min}$. Transfer the callus to the co-cultivation medium. 
D. Selection and regeneration of transgenic plant

1. Rinse the calli 5-6 times with $160 \mathrm{mg} / \mathrm{L}$ timentin solution and dry the calli on sterilized filter paper for $5 \mathrm{~min}$.

2. Transfer the dried calli onto resting media for 10 days at $24^{\circ} \mathrm{C}$ in the dark.

3. After 10 days of resting, transfer the calli to the selection medium for 2 weeks at $25{ }^{\circ} \mathrm{C}$ in the dark.

4. Remove brown and black calli from the selection medium and transfer healthy calli to the fresh section media for the second selection.

5. Incubate the selected calli at $25^{\circ} \mathrm{C}$ under low-light intensity (Normal plant growth condition is approx.130-150 $\mu \mathrm{E} \mathrm{m}^{-2} \mathrm{~s}^{-1}$ light intensity, but in this step, cover the calli with one sheet of white paper for reduced light intensity).

6. After 10 days on the second selection, transfer healthy calli onto regeneration medium at $25^{\circ} \mathrm{C}$ under $16 \mathrm{~h}$ light $/ 8 \mathrm{~h}$ dark condition.

7. Every 10-day transfer calli to fresh regeneration medium up to 6 weeks until roots and shoots are observed.

8. Continue transferring calli and plantlets to fresh selection media for shoot regeneration.

9. Transfer shoot regenerated plantlets to Magenta vessels having MS media without any hormone.

E. Analysis of the regenerated transgenic callus and plants

1. GFP expression test.

a. GFP expression is detected in the callus 3 days after co-cultivation with Agrobacterium, using a Zeiss Axio scope A1 (Carl Zeiss Microscopy, LLC USA).

b. Set-up the microscope with GFP filter, $470 / 40 \mathrm{~nm}$ excitation filter.

c. Observe at least 20 individual callus under the microscope and count GFP expressed cell number.

2. GUS activity assay with leaf segment.

a. Cut the $1 \mathrm{~cm}$ leaf of each regenerated plantlet from the root generation media (Magenta vessels) and place the leaf fragments into the microplate with $100 \mu \mathrm{X}$-Gluc solution.

b. Incubate the plate at $37^{\circ} \mathrm{C}$ overnight (color to fully develop).

c. After incubation, remove the chlorophyll in leaf fragments by replacing X-gluc with $70 \%$ ethanol under the light until the green color fades.

d. The blue-stained product is visible.

\section{Data analysis}

1. Callus induction frequency on the mature embryos

a. Three different genotypes (Triticum aestivum L. cv Chinese Spring, Triticum aestivum L. cv Amidon, and Triticum aestivum L. cv Choteau) of spring wheat examine for callus induction and plant regeneration. 
b. $50-60 \mathrm{~s}$ of each genotype examine callus induction frequency in each replication.

c. After 2 weeks on the callus generation media, determine callus induction rate by size of the callus on the embryos and callus development monitor for 4 weeks.

d. Take a picture of the callus generation plates and measure the longest length of callus by using the ImageJ program.

e. Determine callus induction by over $5 \mathrm{~mm}$ of callus length. And shorter than $5 \mathrm{~mm}$ of callus keep growing on the callus generation media for 2 more weeks.

f. Callus induction frequency calculate as the number of calli generated embryo divide by the total number of embryos on the plate multiply 100 .

Frequency $=\frac{\text { Number of callus generated embryo }}{\text { Total number of embryos of each genotype }} \times 100$

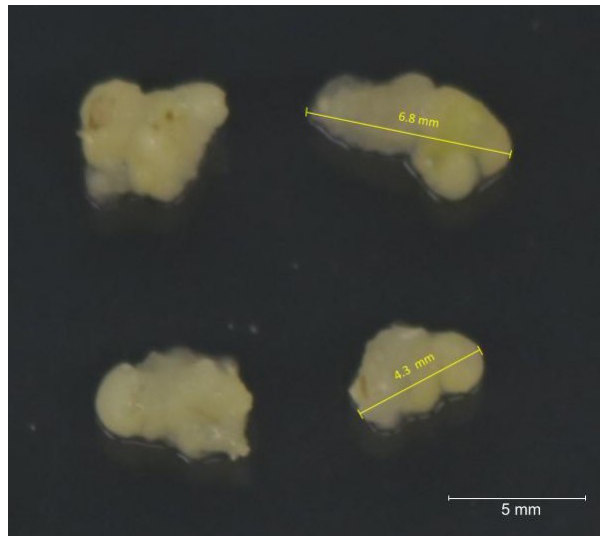

Figure 3. Measure the length of the callus. The yellow line measures the most extended length of the calli using the Image J program, and the scale bar is $5 \mathrm{~mm}$. Measure the callus size for determining the callus generation frequency.

2. The efficiency of the transgenic plant regeneration

a. After 4 weeks from the co-cultivation, start appearing a green tissue on the surface of the callus and generating a shoot on the green tissue.

b. When a shoot generates on the callus, individual shoots separate from the regeneration calli and place in a Magenta vessel having MS media without growth hormone.

c. Count the number of shoots out of the total number of transformed callus.

d. Shoot generation rate is

$$
\frac{\text { Number of shoots }}{\text { Total number of trnasformed callus }} \times 100
$$

e. Keep growing for 2 weeks more and count the number of shoots and calculate the final shoot generation rate.

f. After 4 weeks in the Magenta vessels, count the root induction rate.

g. Root induction rate is 


\section{$\frac{\text { Number of roots induced shoot }}{\text { Total number of trnasformed callus }} \times 100$}

h. The root induction rate is the same as the plant regeneration rate.

\section{$\underline{\text { Recipes }}$}

1. MG media

$5 \mathrm{~g} / \mathrm{L}$ Mannitol

$1 \mathrm{~g} / \mathrm{L}$ L-glutamic acid

$2.5 \mathrm{~g} / \mathrm{L}$ Yeast extract

$5 \mathrm{~g} / \mathrm{L}$ tryptone

$250 \mathrm{mg} / \mathrm{L} \mathrm{KH}_{2} \mathrm{PO}_{4}$

$100 \mathrm{mg} / \mathrm{L} \mathrm{NaCl}$

$100 \mathrm{mg} / \mathrm{L} \mathrm{MgSO}_{4} \cdot 7 \mathrm{H}_{2} \mathrm{O}$

$\mathrm{pH} 7.0$

Add supplement after autoclaving

$1 \mu \mathrm{g} / \mathrm{L}$ Biotin

$25 \mathrm{mg} / \mathrm{L}$ Streptomycin

$10 \mathrm{mg} / \mathrm{L}$ Rifampicin

$50 \mathrm{mg} / \mathrm{L}$ Kanamycin

2. Agrobacterium resuspension medium

$4.3 \mathrm{~g} / \mathrm{L}$ MS salts

$30 \mathrm{~g} / \mathrm{L}$ maltose

$3 \mathrm{~g} / \mathrm{L} \mathrm{KCl}$

$4 \mathrm{~g} / \mathrm{L} \mathrm{MgCl} 2$

$150 \mu \mathrm{M}$ Acetosyringone

$\mathrm{pH} 5.2$

3. Co-cultivation media

$4.3 \mathrm{~g} / \mathrm{L}$ MS basal salt

$1 \mathrm{~g} / \mathrm{L}$ Casein hydrolysate

$350 \mathrm{mg} / \mathrm{L}$ Myo-inositol

$690 \mathrm{mg} / \mathrm{L}$ Proline

$1 \mathrm{mg} / \mathrm{L}$ Thiamine $\mathrm{HCl}$

$5 \mathrm{pM}$ copper (II) sulfate

$150 \mu \mathrm{M}$ Acetosyringone

$0.7 \%$ Phytagel

$\mathrm{pH} 5.8$

4. Callus generation media 
$4.3 \mathrm{~g} / \mathrm{L}$ MS basal salt

$1 \mathrm{~g} / \mathrm{L}$ Casein hydrolysate

350 mg/L Myo-inositol

$690 \mathrm{mg} / \mathrm{L}$ Proline

$1 \mathrm{mg} / \mathrm{L}$ Thiamine $\mathrm{HCl}$

5 pM Copper(II) sulfate

$2.5 \mathrm{mg} / \mathrm{L} \mathrm{2,4-D}$

$0.7 \%$ Phytagel

$\mathrm{pH} 5.8$

5. Resting media

$4.3 \mathrm{~g} / \mathrm{L} \mathrm{MS}$ basal salt

$1 \mathrm{~g} / \mathrm{L}$ Casein hydrolysate

350 mg/L Myo-inositol

$690 \mathrm{mg} / \mathrm{L}$ Proline

$1 \mathrm{mg} / \mathrm{L}$ Thiamine $\mathrm{HCl}$

5 pM Copper(II) sulfate

$2.5 \mathrm{mg} / \mathrm{L}$ 2,4-D

$160 \mathrm{mg} / \mathrm{L}$ Timetine

$0.7 \%$ Phytagel

$\mathrm{pH} 5.8$

6. Selection media

$4.3 \mathrm{~g} / \mathrm{L}$ MS basal salt

$1 \mathrm{~g} / \mathrm{L}$ Casein hydrolysate

350 mg/L Myo-inositol

$690 \mathrm{mg} / \mathrm{L}$ Proline

$1 \mathrm{mg} / \mathrm{L}$ Thiamine $\mathrm{HCl}$

5 pM Copper(II) sulfate

$160 \mathrm{mg} / \mathrm{L}$ Timetine

$2 \mathrm{mg} / \mathrm{L}$ Lipoic acid

$1 \mu \mathrm{M}$ IAA

$25 \mathrm{mg} / \mathrm{L}$ Hygromycin

$0.7 \%$ Phytagel

$\mathrm{pH} 5.8$

7. Regeneration media

$4.3 \mathrm{~g} / \mathrm{L}$ MS basal salt

$1 \mathrm{~g} / \mathrm{L}$ Casein hydrolysate

$350 \mathrm{mg} / \mathrm{L}$ Myo-inositol

$690 \mathrm{mg} / \mathrm{L}$ Proline

$1 \mathrm{mg} / \mathrm{L}$ Thiamine $\mathrm{HCl}$ 


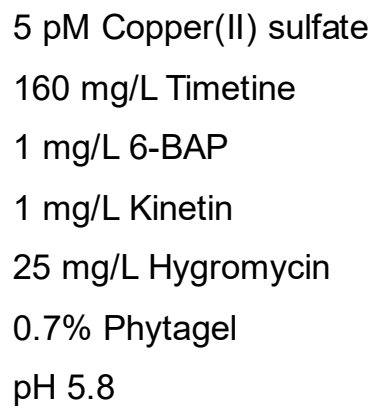

\section{Acknowledgments}

This work was supported by a grant from the Montana Wheat and Barley Committee.

\section{Competing interests}

The authors declare no competing interest.

\section{References}

1. Alikina, O., Chernobrovkina, M., Dolgov, S. and Miroshnichenko, D. (2016). Tissue culture efficiency of wheat species with different genomic formulas. Crop Breed Appl Biotechnol 16(4): 307-314.

2. Bhalla, P. L. (2006). Genetic engineering of wheat--current challenges and opportunities. Trends Biotechnol 24(7): 305-311.

3. Ding, L., Li, S., Gao, J., Wang, Y., Yang, G. and He, G. (2009). Optimization of Agrobacteriummediated transformation conditions in mature embryos of elite wheat. Mol Biol Rep 36(1): 2936.

4. Vasil, V., Castillo, A. M., Fromm, M. E. and Vasil, I. K. (1992). Herbicide resistant fertile transgenic wheat plants obtained by microprojectile bombardment of regenerable embryogenic callus. Nature Biotechnol 10(6): 667-674. 